\title{
HETEROGENEOUS AMORPHIZATION OF P AND As IMPLANTED GaAs AT LOW TEMPERATURES
}

\author{
J. KRYNICKI, H. RzEWUSKI \\ Institute of Nuclear Chemistry and Technology \\ Dorodna 16, 03-195 Warszawa, Poland
}

AND A. Turos

Institute of Nuclear Studies, Hoża 69, 00-681 Warszawa, Poland

\begin{abstract}
Amorphization of $\mathrm{P}$ and As implanted GaAs at liquid nitrogen temperature has been investigated. The post-implantation damage was measured by means of Rutherford Backscattering (RBS) $\mathrm{He}^{+}$channeling technique. The critical dose and critical energy densities for amorphization were determined. From the results obtained it is concluded that for both ions the amorphization process can be satisfactorily described by the heterogeneous model.
\end{abstract}

PACS numbers: $61.80 . \mathrm{Jh}, 61.70 . \mathrm{Tm}$

\section{Introduction}

Many experimental results on the crystalline to amorphous transformation show that the amorphous transition at low implantation temperatures depends primarily on the properties of a crystal than on the implanted ion.

Various ideas have been proposed to explain the nature of a damage cluster or a cascade centered about the ion path [1-5]. With the increase of the implanted dose the damage starts to increase and at sufficiently high dose and low enough temperature a uniform amorphous layer is produced.

Two presently accepted phenomenological models describe this amorphous transition - the spike model (heterogeneous) and the critical energy density model (homogeneous) [6].

The aim of this paper is to check the validity of these models for amorphization induced by "light" and "medium" $\mathrm{P}$ and As implantation of GaAs at low temperature. 


\section{Experimental}

$N$-type $2 \times 10^{17} \mathrm{~cm}^{-3}$ Te doped (100) oriented GaAs crystals (Hoboken Chemicals), cut and polished in a standard way, were implanted in a nonchanneling direction with $\mathrm{P}$ and $\mathrm{As}$ ions close to liquid nitrogen temperature. The fluencies used ranged from $2 \times 10^{13}$ to $2.5 \times 10^{14} \mathrm{~cm}^{-2}$ and from $5 \times 10^{12}$ to $5 \times 10^{13} \mathrm{~cm}^{-2}$ for $\mathrm{P}$ and As ions, respectively, in the same energy range from 40 to $160 \mathrm{keV}$ for both ions. The ion beam intensity was lower than $0.1 \mu \mathrm{A} \mathrm{cm} \mathrm{c}^{-2}$ to minimize the heating of the target. The temperature of the samples was monitored by a copper-constantan thermocouple and kept below $120 \mathrm{~K}$.

Post-implantation disorder distributions'were measured by the RBS channeling technique of $2 \mathrm{MeV} \mathrm{He}{ }^{+}$ions. The analysing $\mathrm{He}$ beam intensity kept below $30 \mathrm{nA}$ made it possible to maintain a low background of RBS spectra.

The measurements were performed on the ion accelerator "Lech" in the Institute of Nuclear Studies (Warsaw).

The depth damage distribution (profiles) were obtained using the classical relation [7].

The computer code TRIM- 89 was used to calculate the critical energy density for each $\mathrm{P}$ and As ion energy.

\section{Results and discussion}

The RBS spectra from the implanted layers of GaAs for various energies and doses of implanted $P$ and As ions have been measured. Using the well-known formula [7] and special computer program "Spectr" [8] the depth damage distributions were calculated.

The critical dose necessary to make crystalline-to-amorphous transformation in GaAs for each $P$ and As ion energy, was determined. In Table I the critical

TABLE I

The critical dose values for $P$ and As ion implantations performed at $120 \mathrm{~K}$.

\begin{tabular}{c|cc|cc}
\hline \hline Energy(KeV) & $\begin{array}{c}\text { Critical dose for P } \\
\left(\times 10^{13} \mathrm{~cm}^{-2}\right)\end{array}$ & \multicolumn{2}{|c}{$\begin{array}{c}\text { Critical dose for As } \\
\left(\times 10^{13} \mathrm{~cm}^{-2}\right)\end{array}$} \\
\hline & $T=120 \mathrm{~K}$ & $T=300 \mathrm{~K}$ & $T=120 \mathrm{~K}$ & $T=300 \mathrm{~K}$ \\
40 & 30 & 120 & 15 & 18 \\
80 & 9 & 55 & 6 & 7 \\
120 & 7 & - & 2.5 & 4.5 \\
160 & 4 & 35 & 1.2 & -
\end{tabular}

dose values are listed for $\mathrm{P}$ and As ion implantations performed at $120 \mathrm{~K}$ and for comparison at room temperature taken from the previous paper [8]. We note that the critical dose values decrease with the energy increase of the implanted ions independently from their mass and temperature of implantation. Analy'sis 
of the reported data has been performed and combined with the concepts from homogeneous and heterogeneous models of amorphization.

From TRIM-89 calculations depth distributions of deposited energy in the crystal per implanted $\mathrm{P}$ and As ions have been obtained. Comparing these values with the critical dose estimated from the experiment, the critical energy density (CED) for each energy of implantation was found (Table II).

TABLE II

Critical energy density (CED) for $\mathrm{P}$ and As.

\begin{tabular}{c|c|c}
\hline \hline Energy (keV) & \multicolumn{2}{|c}{ CED $\left(10^{20} \mathrm{keV} \mathrm{cm}^{-3}\right)$} \\
\hline & $\mathrm{P}$ & As \\
40 & 7 & 8.4 \\
80 & 4 & 3.6 \\
120 & 3 & 1.5 \\
160 & 1.4 & 1.1
\end{tabular}

In the case of homogeneous model of amorphization the calculated CED values should be approximately constant with ion energy which does not occur in our case.

For $\mathrm{P}$ ions CED differs by a factor of 5 for 40 and $160 \mathrm{keV}$ and for As ions by a factor of 7.5 for the same energy range.

These results suggest that the amorphization transition cannot be described by the homogeneous model. The interpretation of the results obtained using the heterogeneous model of amorphization proposed by Gibbons [1] involves the overlap of damage clusters. The area fraction of $A_{\mathrm{A}}$ of amorphous material is given by

$$
A_{\mathrm{A}}=1-\sum_{k=0}^{m} \frac{\left(A_{i} \Phi\right)^{k}}{k !} \exp \left(-A_{i} \Phi\right)
$$

where $A_{i}$ is the damage created by a single ion, $\Phi-$ a dose of implanted ions and $m$ - the number of ions required to damage the same region to produce amorphization.

Figures 1 and 2 show the experimental data for amorphous build-up with the dose at various implantation energies for $\mathrm{P}$ and As ions superimposed on theoretical curves (solid lines) from Eq. (1) for $m=1$ and $m=2$.

Since the agreement for ions having various masses is quite good in character it can be assumed that the heterogeneous model is more satisfactory to describe the amorphization process in GaAs. 


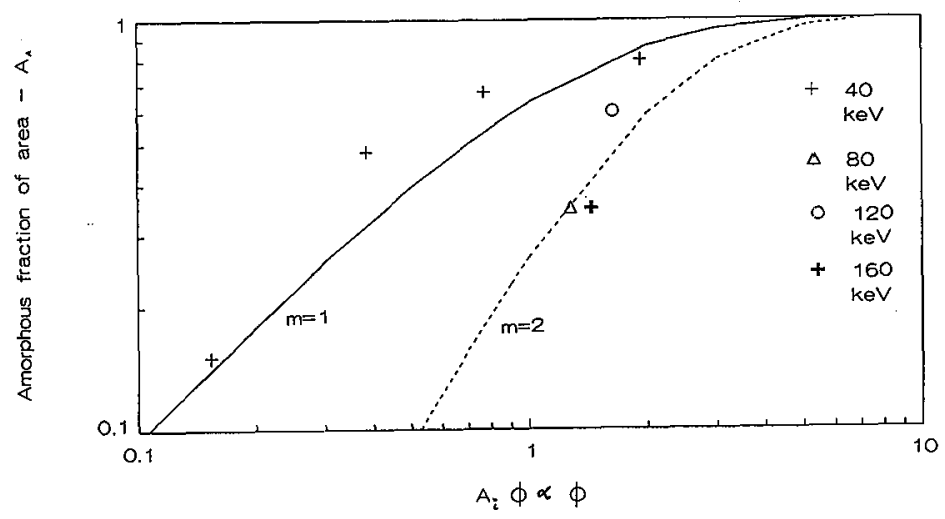

Fig. 1. Fit to overlap model [1] for P implanted GaAs at $120 \mathrm{~K}$.

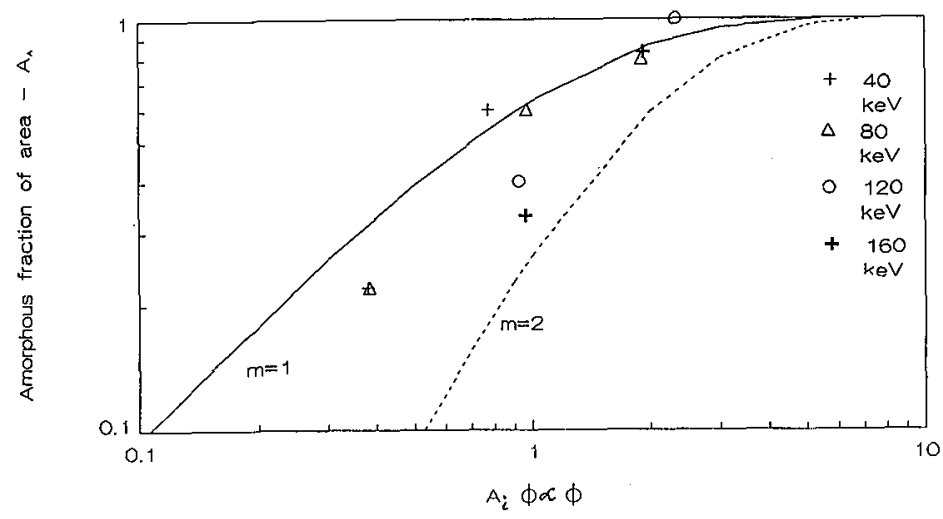

Fig. 2. Fit to overlap model [2] for As implanted GaAs at $120 \mathrm{~K}$.

\section{Summary}

In the present study amorphization of GaAs implanted with $\mathrm{P}$ and As ions at $120 \mathrm{~K}$ has been investigated. RBS channeling technique was used to measure the depth profile of post-implantation defects. The critical doses for $\mathrm{P}$ and As ions for various energies have been found and compared with those for room temperature [8].

The critical energy density (CED) values have also been determined being not constant with ion energy. Using Gibbons' relation [1] the experimental build-up with various implantation energies for both ions was calculated and compared with theoretical curves for $m=1$ and $m=2$ overlaps. It is concluded that the amorphization process for $\mathrm{P}$ and As implanted GaAs at $120 \mathrm{~K}$ is better described by the heterogeneous model. 


\section{References}

[1] J.F. Gibbons, Proc. IEEE 60, 1062 (1972).

[2] F. Seitz, J.S. Kochler, Solid State Physics, Vol. 2, Academic Press, New York 1956, p. 356.

[3] J.A. Brinkman, Am. J. Phys. 24, 246 (1956).

[4] A. Seeger, Proc. Internat. Conf. on Peaceful Use of Atomic Energy 250 (1958).

[5] L. Chaddertonn, F.H. Eisen (Eds.), Proc. 1st Conf. Ion Implantation, Gordon and Breach, New York 1971, p. 445.

[6] J.R. Dennis, E.B. Halle, J. Appl. Phys. 49(3), 119 (1978).

[7] W.K. Chu, J.W. Mayer, M.A. Nicolet, Backscattering Spectroscopy, Academic Press, New York 1978.

[8] J. Krynicki, H. Rzewuski, A. Turos, R. Sidor, to be published in Radiation Effects and Defects in Solids. 